\title{
Surface fitting in geomorphology - Examples for regular-shaped volcanic landforms
}

\author{
M. Favalli a,*, D. Karátson ${ }^{\text {b }}$, J. Yepes ${ }^{\text {c }}$, L. Nannipieri ${ }^{\text {a }}$ \\ ${ }^{a}$ Istituto Nazionale di Geofisica e Vulcanologia, via della Faggiola 32, I-56126 Pisa, Italy \\ ${ }^{b}$ Department of Physical Geography, Eötvös University, Pázmány s. $1 / \mathrm{C}, \mathrm{H}-1117$ Budapest, Hungary \\ ${ }^{c}$ Department of Civil Engineering, University of Las Palmas de Gran Canaria, 35017 Canary Islands, Spain
}

\begin{abstract}
A B S T R A C T
In nature, several types of landforms have simple shapes: as they evolve they tend to take on an ideal, simple geometric form such as a cone, an ellipsoid or a paraboloid. Volcanic landforms are possibly the best examples of this "ideal" geometry, since they develop as regular surface features due to the point-like (circular) or fissure-like (linear) manifestation of volcanic activity.

In this paper, we present a geomorphometric method of fitting the "ideal" surface onto the real surface of regularshaped volcanoes through a number of case studies (Mt. Mayon, Mt. Somma, Mt. Semeru, and Mt. Cameroon). Volcanoes with circular, as well as elliptical, symmetry are addressed. For the best surface fit, we use the minimization library MINUIT which is made freely available by the CERN (European Organization for Nuclear Research). This library enables us to handle all the available surface data (every point of the digital elevation model) in a onestep, half-automated way regardless of the size of the dataset, and to consider simultaneously all the relevant parameters of the selected problem, such as the position of the center of the edifice, apex height, and cone slope, thanks to the highly performing adopted procedure.

Fitting the geometric surface, along with calculating the related error, demonstrates the twofold advantage of the method. Firstly, we can determine quantitatively to what extent a given volcanic landform is regular, i.e. how much it follows an expected regular shape. Deviations from the ideal shape due to degradation (e.g. sector collapse and normal erosion) can be used in erosion rate calculations. Secondly, if we have a degraded volcanic landform, whose geometry is not clear, this method of surface fitting reconstructs the original shape with the maximum precision. Obviously, in addition to volcanic landforms, this method is also capable of constraining the shapes of other regular surface features such as aeolian, glacial or periglacial landforms.
\end{abstract}

\section{Introduction}

Fitting parametric surfaces (e.g. Hermann et al., 1997; Ahn, 2008) is widely applied in many scientific fields. Surface fitting is used to find an ideal, simple surface that is closest to the real surface. This is helpful to know whether, and to what extent, the studied form is regular, and/or how it can be reconstructed. Fitting is done by defining the surface that minimizes the distance between the ideal and real surfaces. In geomorphometry, the "ideal" surface is considered a regular, geometric form (Evans, 1990; Deng, 2007; Pike et al., 2009; Gercek et al., 2011) having the shape of a plane, cone, ellipsoid, hyperboloid or paraboloid. These forms have a simple mathematical expression: they are described by equations $f(x, y, z)=0$ where the function $f$ has a simple algebraic expression.

\footnotetext{
* Corresponding author. Tel.: +39050 8311948; fax: +39050 8311942. E-mail address: favalli@pi.ingv.it (M. Favalli).
}

On Earth, a number of geomorphic elements show a regular shape, including small-scale landforms such as cirques, drumlins, sand dunes, alluvial fans, dolines, polygonal soils and cracks, columnar jointing, and scoria cones, to large-scale landforms such as composite and shield volcanoes (e.g. Pike et al., 2009). These simple geometric shapes close to "ideal" ones are perhaps best represented by volcanic landforms. Indeed, many of them tend to develop as regular surface features (cf. Wood, 1980; Thouret, 1999; Davidson and da Silva, 2000; Karátson et al., 2010; Fornaciai et al., 2012) controlled by the point-like (i.e. circular) or fissure-like (i.e. linear) nature of eruptive activity. For example, the surprisingly regular, simple stratovolcanoes can grow up over several thousands of years, with their minor shape differences reflecting various styles of volcanic activity (Karátson et al., 2010). The fact that the shape of such volcanoes follows a regular geometry is highly useful when inferring the original configuration of a degraded volcanic edifice, e.g. affected by sector collapse or intense erosion.

In this paper, we present the methodology for identifying and fitting an ideal shape to a real surface through case studies on a selection of various volcanic landforms. Our goal is to see if, and to what extent, a 
regular geometric shape can be fitted to apparently simple volcanic constructs, and how the computation procedure can be best performed. We do not focus only on the most regular examples as in one of our previous approaches (Karátson et al., 2010), but investigate how surface fitting can be done to any simple volcano, including less symmetric stratovolcanoes and shield volcanoes.

For a successful surface fit, a method or code that minimizes the function of several variables is needed. Up to now such minimization problems in geomorphology have been dealt with by splitting the complex problem into simpler problems, which are easier to manage. For example when trying to fit a conical shape to a stratovolcano, one usually starts by finding the center of radial symmetry of the real edifice, then the best fitting profile (e.g. Cioni et al., 1999; Karátson et al., 2010). In many complex cases, splitting the problem into easier steps is very difficult and often done at the expense of the fit accuracy.

Here, we use the minimization library MINUIT available from CERN (European Organization for Nuclear Research) to solve complicated cases without having to resort to splitting the problem. By using MINUIT, we can directly find all the required parameters for the best fitting surface to any real surface. Using MINUIT allows us to:

1. handle all the available data (every point of the DEM) regardless of the size of the dataset; and

2. consider simultaneously all the relevant parameters of the selected problem, such as the position of the center of the edifice, apex height, and slope of the cone, thanks to the highly performing procedure; and select the weight for every single point in the minimization depending on the criteria required by the specific problem.

In this paper, we present the methodological background of surface fitting, and a number of case studies on selected volcanoes; then we discuss the applicability and advantages of the method in general.

\section{Methodology}

\subsection{The MINUIT package}

MINUIT was conceived as a tool to find the minimum value of a multi-parameter function and analyze the shape of the function around this minimum. It was initially written in FORTRAN during 1975-1989 at CERN by Fred James (CN/ASD Group, 1993). MINUIT is a platform independent package created in an object-oriented way using standard $\mathrm{C}++$ and it is independent of any external package. It can be freely downloaded at www.cern.ch/minuit.

The MINUIT package acts on a multiparameter 'objective function' called the FCN. This function is usually $\chi^{2}$ (chi-squared), but could also be other mathematical functions (James and Winkler, 2004). The package can also do histogramming, data handling and graphics, but these functions are not used in this paper.

As an example, let us consider $n$ data points $\left(x_{i}, m_{i}\right)$ where the independent $y$ measurement $m_{i}$ has an individual uncertainty $o_{i}$. Our goal is to model $y$ as a function $y=f\left(x_{i}, \alpha\right)$ where $\alpha$ is an $N$-dimensional vector of coefficients that must be determined. If the uncertainties in the data describe errors that may be approximated as zero-mean Gaussian random variables, then Gaussian estimation may be used to evaluate the coefficients $\alpha$, and $\chi^{2}$ minimization is the optimal method to apply. The $\chi^{2}$ distribution with $n$ degrees of freedom is by definition the sum of the squares of $n$ independent standard normal variables. In our case, for uncorrelated errors, $\chi^{2}$ is the sum of the ratios of the squared differences between the model and the observed values divided by the uncertainty variance (James and Winkler, 2004):

$\chi^{2}(\alpha)=\sum_{i=1}^{n} \frac{\left[f\left(x_{i}, \alpha\right)-m_{i}\right]^{2}}{o_{i}^{2}}$

where $i$ spans over all the $n$ data points $\left(x_{i}, m_{i}\right)$ with $m_{i}$ the independent $y$ measurements, and $\sigma_{i}$ are the uncertainty of the measurement $m_{i}$.
Since MINUIT is commonly used to perform fits to experimental data, the parameter errors can be calculated and will be proportional to the uncertainty in the data.

\subsection{Surface fitting}

We fit ideal surfaces to a digital elevation model (DEM) by minimizing the root mean square error (RMSE):

$R M S E=\sqrt{\frac{\sum_{i, j}\left[H_{i, j}-f\left(x_{i}, y_{j}, \alpha\right)\right]^{2}}{n}}$

where the integers $i$ and $j$ span the row and column positions of the DEM grid; $x_{i}, y_{j}$ and $H_{i j}$ are the $x, y$ and $z$ coordinate positions, respectively, of the DEM point identified by $i$ and $j$; $n$ is the total number of DEM points; and $f$ is the fitting function.

Eq. (2) has the same local minima as the corresponding $\chi^{2}$ in Eq. (1) where we assign the same error to every DEM data point, so both formulations should converge on the same result. While $\chi^{2}$ values are directly dependent on the number of data points, RMSE is not, and thus allows an easy comparison between minimizations performed on domains with different numbers of points, due either to different domain extensions or different DEM resolutions (Table 1 ).

We can also introduce different weights $\left(\omega_{i, j}\right)$ for the different points in the minimization procedure, by reintroducing the role of $\sigma_{i}$ in Eq. (1), to calculate a weighted value of RMSE:

$R M S E=\sqrt{\frac{\sum_{i, j} \omega_{i, j}\left[H_{i, j}-f\left(x_{i}, y_{j}, \alpha\right)\right]^{2}}{\sum_{i, j} \omega_{i, j}}}$.

This is useful to give emphasis to different portions of a volcanic edifice. The minimization procedure needs a starting point (a guess, i.e. an initial value for the free parameters) and will converge to the connected local minimum. In general, different input values might converge to different local minima so some caution is required when assigning input values for the parameters. However, in all the presented cases, the choice of initial values was irrelevant when determining the minimum as long as the initial values were chosen from within the physical range of the parameters. In other words, any DEM point inside the edifice is an adequate initial point for the center of the edifice and will converge to the "true" center. Although the $\mathrm{C}++$ implementation of MINUIT currently has no limit on the total number $N$ of the free parameters being fitted, James and Winkler (2004) concluded that, practically, it is limited to a maximum number of 15 parameters at a time. The most complex case we present here involved the simultaneous determination of only eight free parameters.

\subsection{Study areas}

Surface fitting methodology will be shown in detail through a number of case studies: Mt. Mayon, Mt. Semeru, Mt. Somma, and Mt. Cameroon, which have been selected as examples with specific geometry. Stratovolcanoes have long been recognized as representing regular surface features on Earth. The most regular-shaped stratovolcanoes have extremely high circularity (as high as 0.94 to 0.97 for the 20 most symmetric terrestrial examples; Karátson et al., 2010). Among these, a prominent one, Mayon volcano in the Philippines (Ramos-Villarta et al., 1985; Castillo and Newhall, 2004) is an almost perfect "textbook" stratovolcano, whose surface is ideal for fitting.

Semeru volcano is the highest mountain on Java (Indonesia) and has been erupting continuously since 1967 , generating vulcanian explosions (Lavigne, 2004). This stratovolcano exhibits a very good circular symmetry but its average profile is slightly different from that of Mayon: while the Mayon profile can be well fitted by an exponential 
Table 1

Surface fit parameters of the studied volcanoes.

\begin{tabular}{|c|c|c|c|c|c|c|c|c|c|c|c|c|}
\hline \multirow[t]{2}{*}{ Volcano } & \multicolumn{2}{|c|}{ Center of cone } & \multirow[t]{2}{*}{ N. pts. } & \multirow[t]{2}{*}{ Fit type $^{a}$} & \multicolumn{2}{|c|}{ Linear profile } & \multicolumn{3}{|c|}{ Exponential profile } & \multirow[t]{2}{*}{$R M S E(\mathrm{~m})$} & \multirow[t]{2}{*}{ Remarks } & \multirow[t]{2}{*}{ Figs. } \\
\hline & $X(\mathrm{~m})$ & $Y(\mathrm{~m})$ & & & $q(\mathrm{~m})$ & $m$ & $a(m)$ & $\mathrm{b}\left(\mathrm{m}^{-1}\right)$ & $c(m)$ & & & \\
\hline \multirow[t]{3}{*}{ Mayon } & 574,246 & $1,465,512$ & 2425 & Lin. & 2324.7 & -0.543 & & & & 44.9 & $z>1000 m$ & $1 b$ \\
\hline & 574,198 & $1,465,556$ & 26,290 & Exp. & & & 2509.4 & $-3.94 \cdot 10^{-4}$ & 67.1 & 34.4 & & $1 \mathrm{~b}$ \\
\hline & 574,242 & $1,465,499$ & 26,290 & Exp. & & & 2486.2 & $-3.83 \cdot 10^{-4}$ & 53.2 & $37.5^{\mathrm{b}}$ & Weight $1 / \mathrm{R}$ & $1 c, 2 b$ \\
\hline \multirow[t]{3}{*}{ Semeru } & 711,731 & $9,103,234$ & 3200 & Lin. & 3715.9 & -0.536 & & & & 64.9 & $z>2000 \mathrm{~m}$ & $3 b$ \\
\hline & 711,636 & $9,103,237$ & 22,587 & Exp. & & & 3395.0 & $-2.46 \cdot 10^{-4}$ & 520.6 & 51.2 & & $3 c$ \\
\hline & 711,651 & $9,103,223$ & 22,587 & $\operatorname{Lin}+\operatorname{Exp}$ & 3719.1 & -0.538 & 3051.4 & $-2.10 \cdot 10^{-4}$ & 436.5 & 47.1 & Mix. fit ${ }^{\mathrm{C}}$ & $3 d$ \\
\hline \multirow[t]{2}{*}{ Somma } & 451,746 & $4,519,713$ & 211,640 & Exp. & & & 2181.2 & $-6.02 \cdot 10^{-4}$ & 58.8 & 39.9 & & $4 d$ \\
\hline & 451,786 & $4,519,766$ & 211,640 & Exp. & & & 1974.5 & $-4.14 \cdot 10^{-4}$ & -76.5 & d & d & $4 c, 4 e$ \\
\hline \multirow[t]{4}{*}{ Cameroon } & 519,321 & $10,465,661$ & 66,625 & Lin. & 3302.1 & -0.151 & & & & 431.9 & & $5 c, 6 a$ \\
\hline & 518,879 & $10,465,178$ & 66,625 & Lin. & 3835.9 & -0.208 & & & & $302.0^{\mathrm{b}}$ & Weight $1 / R$ & $5 \mathrm{~d}$ \\
\hline & 519,131 & $10,465,810$ & 66,625 & Lin. & 3810.1 & -0.154 & & & & 158.7 & Ell. base $\mathrm{e}^{\mathrm{e}}$ & $5 \mathrm{e}, 6 \mathrm{c}$ \\
\hline & 519,206 & $10,465,763$ & 66,625 & Gauss. & & & & & & 116.7 & Sup. base ${ }^{f}$ & $5 \mathrm{f}, 6 \mathrm{e}$ \\
\hline
\end{tabular}

${ }^{a}$ Lin: using Eq. (4); Exp: using Eq. (5); Gauss: using Eq. (9).

${ }^{b}$ RMSE calculated using Ec. (3) and a weight inversely proportional to distance from the center of the volcanic edifice $R$.

${ }^{\mathrm{c}} \mathrm{R}_{d}=3206.9 \mathrm{~m}$ (see main text).

d The function minimized is not RMSE - see main text

e The fitting surface is a cone with an elliptic base. The $m$ parameter refers to the profile along the major axis. Azimuth direction of the ellipse major diameter $=38.5^{\circ}$. Ratio between short and long axes $=0.51$.

${ }^{\mathrm{f}}$ The fitting surface is a cone with Gaussian profile and superelliptic base (Eq. (7)). Azimuth direction of the ellipse major diameter $=39.3^{\circ}$. Ratio between short and long axes $=0.48$.

curve, the upper part of Semeru fits a cone with a simple linear profile (Karátson et al., 2010).

The Somma-Vesuvius volcanic complex (Italy) consists of an older volcano, Mt. Somma, which is truncated by a summit caldera, with a more recent cone, Vesuvius, which has grown with in the caldera during the past 2000 years (Cioni et al., 1999). The old, truncated edifice of Somma volcano, with its highly dissected flanks, is a good example of how surface fitting can help reconstruct the original shape of a volcanic edifice.

Mt. Cameroon (Cameroon, West Central Africa) is an effusive continental volcano with an unusual, elongated shape and a flat top (Mathieu et al., 2011). The volcano has been active during the last few million years (Fitton, 1983; Marzoli et al., 2000), producing fissure eruptions, mostly lava flows, along NE-SW trending rifts that follow the direction of the Cameroon Volcanic Line (Fitton and Dunlop, 1985; Déruelle et al., 2007). It has been chosen as a test case for surface fitting to an overall elongated shape.

\subsection{Digital elevation models}

We used two different topographic datasets, both in the form of grids. We treated each individual grid cell within the area of interest as a separate input data point.

In three of the four examples (Mayon, Semeru and Cameroon), we made use of the 90-m-resolution DEM derived from the Shuttle Radar Topography Mission (SRTM; Rabus et al., 2003). The SRTM dataset have an almost global coverage and can be freely downloaded at http://www2.jpl.nasa.gov/srtm. The 90-m cell size makes it appropriate for morphometric analyses over large areas where the focus is on largescale surface features. The SRTM DEM is well-known and assessed; Rabus et al. (2003) confirmed a vertical absolute accuracy of $\pm 16 \mathrm{~m}$ for $90 \%$ of the data across the entire mission and a relative accuracy of $\pm 6 \mathrm{~m}$ on a local, $50-100-\mathrm{km}$ scale. In our SRTM-based case studies the reconstructed volcanic edifices have radii varying between 10 and $20 \mathrm{~km}$, and the number of points used for the surface fitting ranges from 2500 to 65,000 (Table 1 ).

In the case study of Mt. Somma we used the TINITALY/01 DEM which is currently the most accurate DEM covering the whole Italian territory (Tarquini et al., 2007). The DEM has been released recently and it is now downloadable for scientific purposes (for details, see Tarquini et al., 2012). Owing to the great heterogeneity in density and accuracy of the input data, the DEM is in a Triangular Irregular Network format. Tarquini et al. (2007) carried out a comprehensive assessment of the accuracy of the DEM, finding RMSE values for elevation between
0.8 and $6.0 \mathrm{~m}$ depending on the region, with an overall RMSE value $<3.5 \mathrm{~m}$. We converted it into a grid form with a step of $10 \mathrm{~m}$. The Mt. Somma reconstruction has a radius of $\sim 5 \mathrm{~km}$ and was carried out using $~ 200,000$ data points.

\section{Results of surface fitting}

\subsection{Mayon}

Using the traditional, "splitting" approach, Karátson et al. (2010) first determined the center of the edifice, then calculated the average radial profile, and finally found the best fitting profile. We performed the surface fitting directly by finding the position of the center of the edifice as well as the radial profile in one step.

We approximated the shape of Mayon edifice in three different ways. We first defined the cone (i.e. the position of the center and the radial profile) that best fits (minimizing Eq. (2)) the portion of the stratovolcano above $1000 \mathrm{~m}$ in elevation (Fig. 1b). For our purpose, fitting a cone to a dataset means finding the $x$ and $y$ positions of the center of the cone, the cone apex elevation, and the slope of the cone flanks: a total of four parameters to be determined. Taking the center as the reference origin, we can express the cone surface as the set of points satisfying the linear relationship:

$z=m R+q$

where $R$ is the planar distance from the origin, $z$ is the elevation and $m$ and $q$ are constants representing the elevation of the apex and the tangent of the slope, respectively.

Then we found the "ideal" conical surface defined by an exponential profile that best fits all the elevation points of the whole volcano, within the domain shown in Fig. 1a. This means that we replace the linear expression of Eq. (4) with the exponential expression:

$z=a \exp (b R)+c$

where $a, b$ and $c$ are constants. In this case the height of the edifice as represented by the fitting surface is $a+c$ (Fig. 1c).

Finally, to give more relevance to the upper part of the volcano, we approached the conical surface with an exponential profile that best fits the DEM, assigning to each point a weighting inversely proportional to its distance from the edifice center using Eq. (3) (Fig. 1d).

The parameter values and the root mean square errors of the three different fits are shown in Table 1 . The resulting errors of a few tens of 

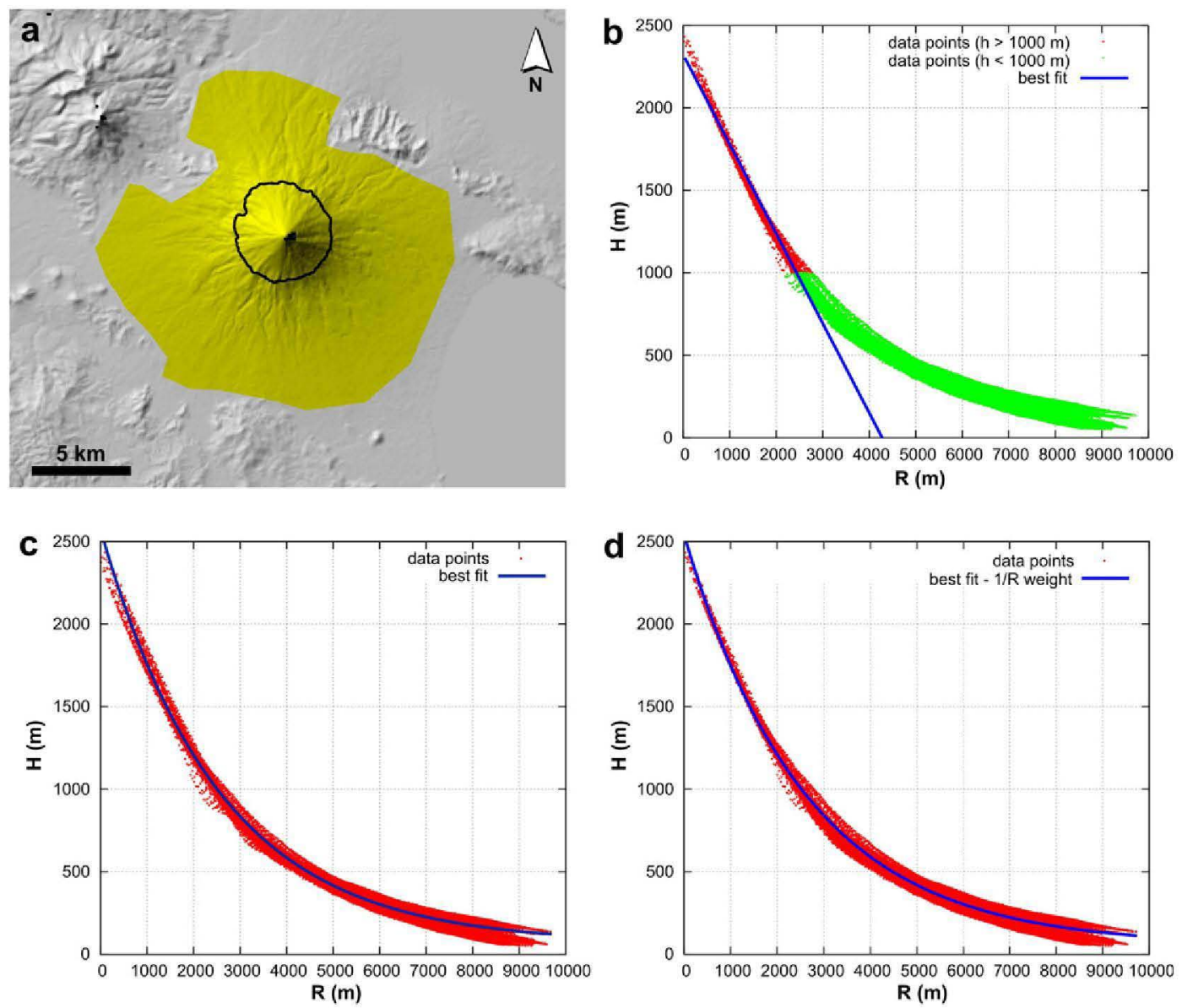

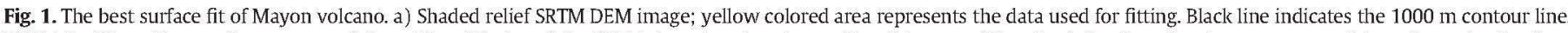

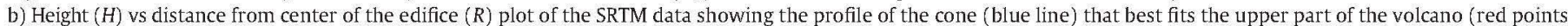

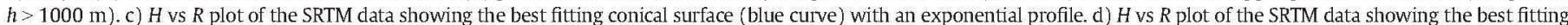

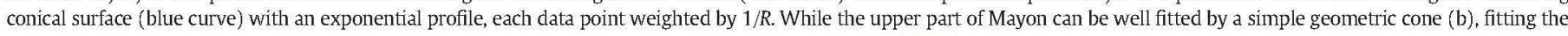

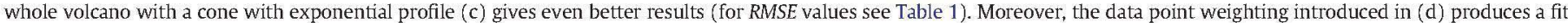
that also approximates the volcano very well in the upper part where the number of data points is small. See main text for further explanations.

meters can be considered as being very low in relation to the $2.5-\mathrm{km}$ high and 18-km wide volcanic edifice.

It is obvious that, in general, the upper part of Mayon stratovolcano ( $h>1000 \mathrm{~m}$ ) can be fitted by a geometric cone (i.e. with a linear profile; Fig. 1b). However, fitting the whole stratovolcano with an exponential profile gives better results (Fig. 1c). The best fit with a geometric cone of the upper part ( $h>1000 \mathrm{~m}$ ) yields an RMSE value of $44.9 \mathrm{~m}$. In contrast, if the whole colored area of Fig. 1a is fitted with a conical surface with an exponential profile (Figs. $1 \mathrm{c}$ and 2 and Table 1), the error is only $34.4 \mathrm{~m}$. Fitting by minimizing RMSE with a weighting proportional to the inverse distance from the center (Eq. (3)) shows that even if the results are numerically worse (RMSE $=37.5 \mathrm{~m}$ as opposed to $34.4 \mathrm{~m}$ of the fit without weightings), they approximate the upper part of the volcano very well (Fig. 1d).

\subsection{Semeru}

This stratovolcano is also amongst the most circular ones on Earth and belongs to the $C$ (conical) type volcanoes according to Karátson et al. (2010). This particular shape is defined, apart from the common exponential lower profile seen for Mayon, by a linear upper profile, i.e. representing a truly conical summit. Therefore, we can fit the upper part (all points closer than radius $R_{d}$ to the edifice center) with a cone with a linear profile, and the lower part with a regular form with an exponential profile (all points further from the edifice center than radius
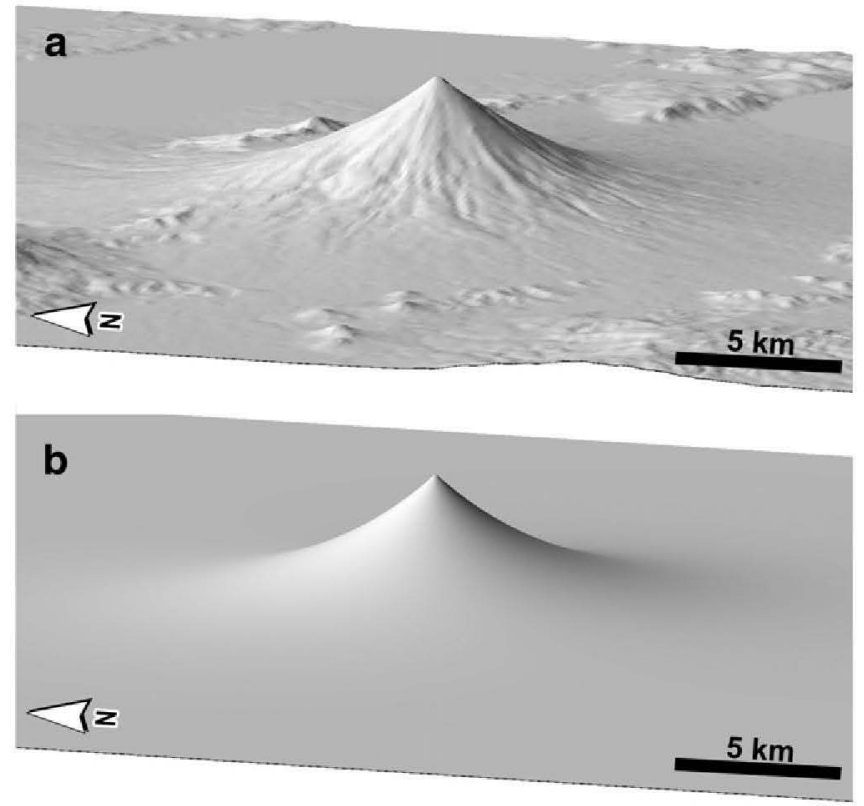

Fig. 2. Oblique view of Mayon volcano viewed from the East. a) SRTM DEM image. b) The best fitting conical surface with an exponential profile (cf. Fig. 1d, Table 1). 
$R_{d}$ ). In this case, the free parameters to be determined by the minimization are the $x$ and $y$ coordinates at the center of the edifice (center of symmetry); $m$ and $q$ for the linear profile; $a, b$ and $c$ for the exponential profile; and $R_{d}$. Ensuring continuity of the profile at $R_{d}$ reduces the total number of free parameters by one. Fig. 3 shows the results of surface fitting to the elevation point cloud. First we fitted the upper part of the volcano ( $h>2000 \mathrm{~m}$ ) with a cone (i.e. linear profile), obtaining an RMSE value of $64.9 \mathrm{~m}$ using only 3200 SRTM DEM points (Fig. 3b). In Fig. 3c, we fitted the yellow region of Fig. 3a with a conical surface with an exponential profile: the resulting RMSE value is $51.2 \mathrm{~m}$, much lower than the cone fit in spite of the significantly higher number of data points fitted (22,587). A mixed fit (i.e. combined exponential and linear profiles) provides a better fit, with an RMSE value of $47.1 \mathrm{~m}$, representing a further improvement of $8 \%$ (Fig. 3d). Karátson et al. (2010) pointed out that these slight deviations between the linear and non-linear profile fits of the upper portion of the volcano are minor but systematic differences between the regular-shaped stratovolcanoes. For example, the same mixed fit, applied to Mayon volcano, would lead to an improvement of only $1 \%$ compared to the fit of a conical surface with simple exponential profile.

\subsection{Mt. Somma}

An example of defining the geometry of an old, truncated edifice is given by Somma volcano, Italy, which does not have a highly regular shape. In a previous approach, without the application of DEMs, Cioni et al. (1999) reconstructed the original shape by using drainage network analysis. Namely, after identifying the extent and shape of the remnant part of Somma edifice, they firstly determined the center of the cone based on radial drainage on the outer slopes, then reconstructed the maximum elevation of the original edifice at around 1600-1900 m. Cioni et al. (1999) postulated a symmetric (although not well-defined) original edifice representing the lower flanks of the polyphase caldera volcano of Somma.

Fig. 4a shows the highly dissected outer flanks of the old $(>18,000 \mathrm{yr})$ Somma volcano selected for analysis. There is a strong contrast between the old surface, the Piano delle Ginestre nested intracaldera center, reconstructed as a tuff cone by Cioni et al. (1999) with a lava flowinundated flat top, and the younger smooth-surfaced cone of Vesuvius. The dissected margins of Piano delle Ginestre, in particular to the SW, raise the possibility that those flanks may have been inherited from the Somma edifice.

Profile analysis of Somma volcano (Fig. 4d) shows that the elevation points around the flanks tend to be evenly distributed, defining a concave shape. In detail, some sectors are located below, others above the average, as shown in Fig. 4a. This gives a minor asymmetry to the circular shape.

In Fig. 4d, the profile of the reconstructed surface fits the data well. However, it is obvious that the envelope of the original surface is even better if the highest elevated points - i.e. ridges - are considered for surface fitting (Fig. 4e). This is because erosion operates mostly or exclusively in the valleys until the planèze stage (cf. Cotton, 1952; Karátson et al., 1999), so the intervening ridges are better in taking the remnant surfaces into account. The higher ridges can be given greater preference
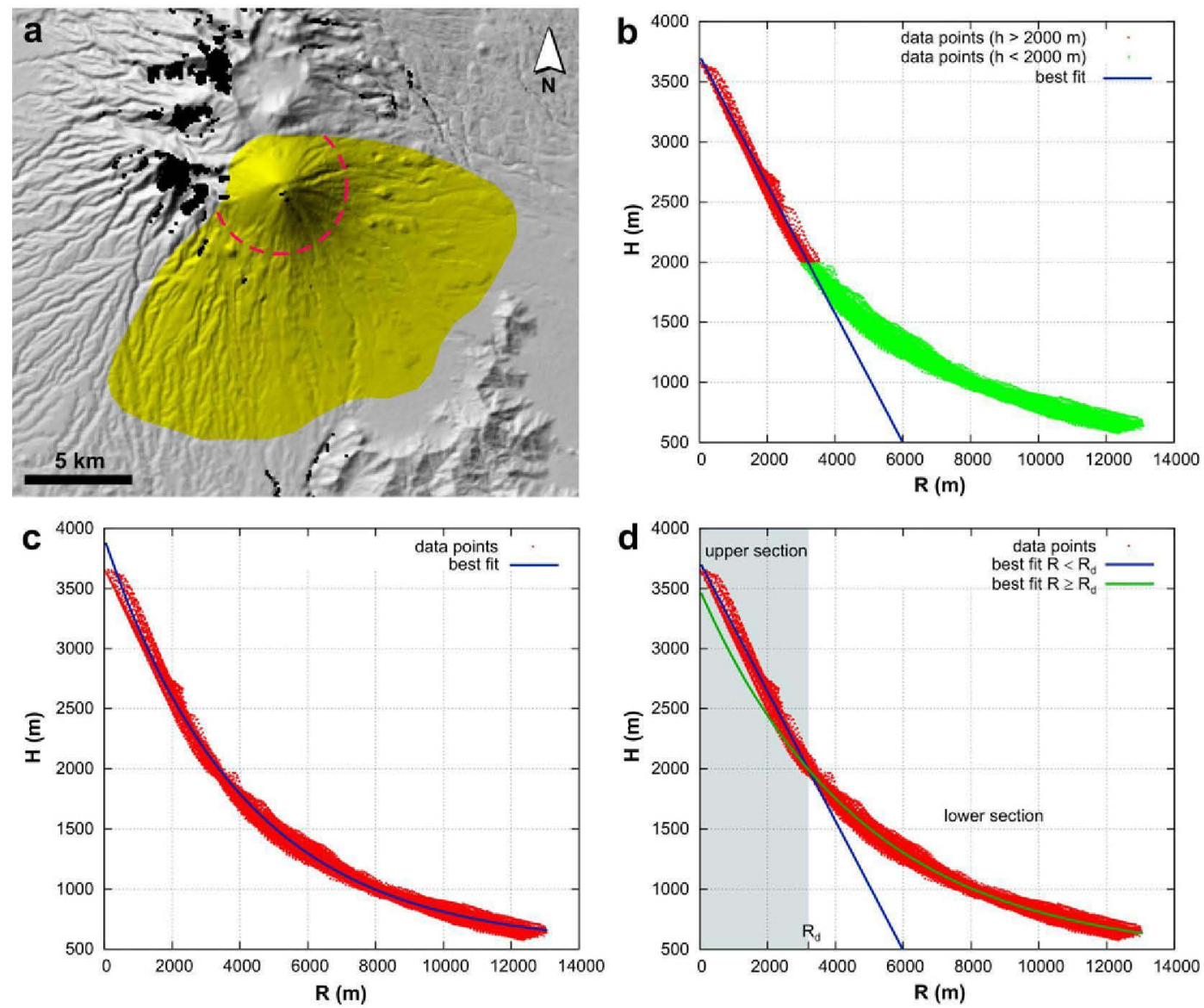

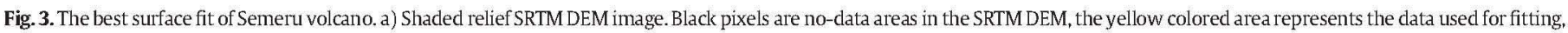

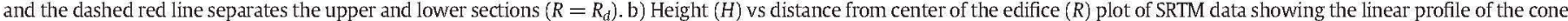

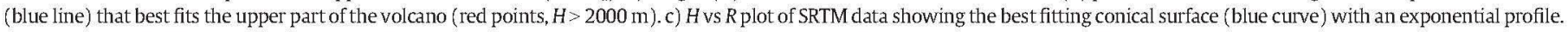

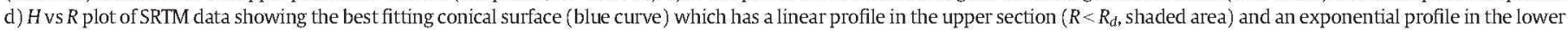
section $\left(R \geq R_{d}\right)$. $R_{d}$ itself was determined by the best fit procedure. See main text for explanation and Table 1 for best fit values. 

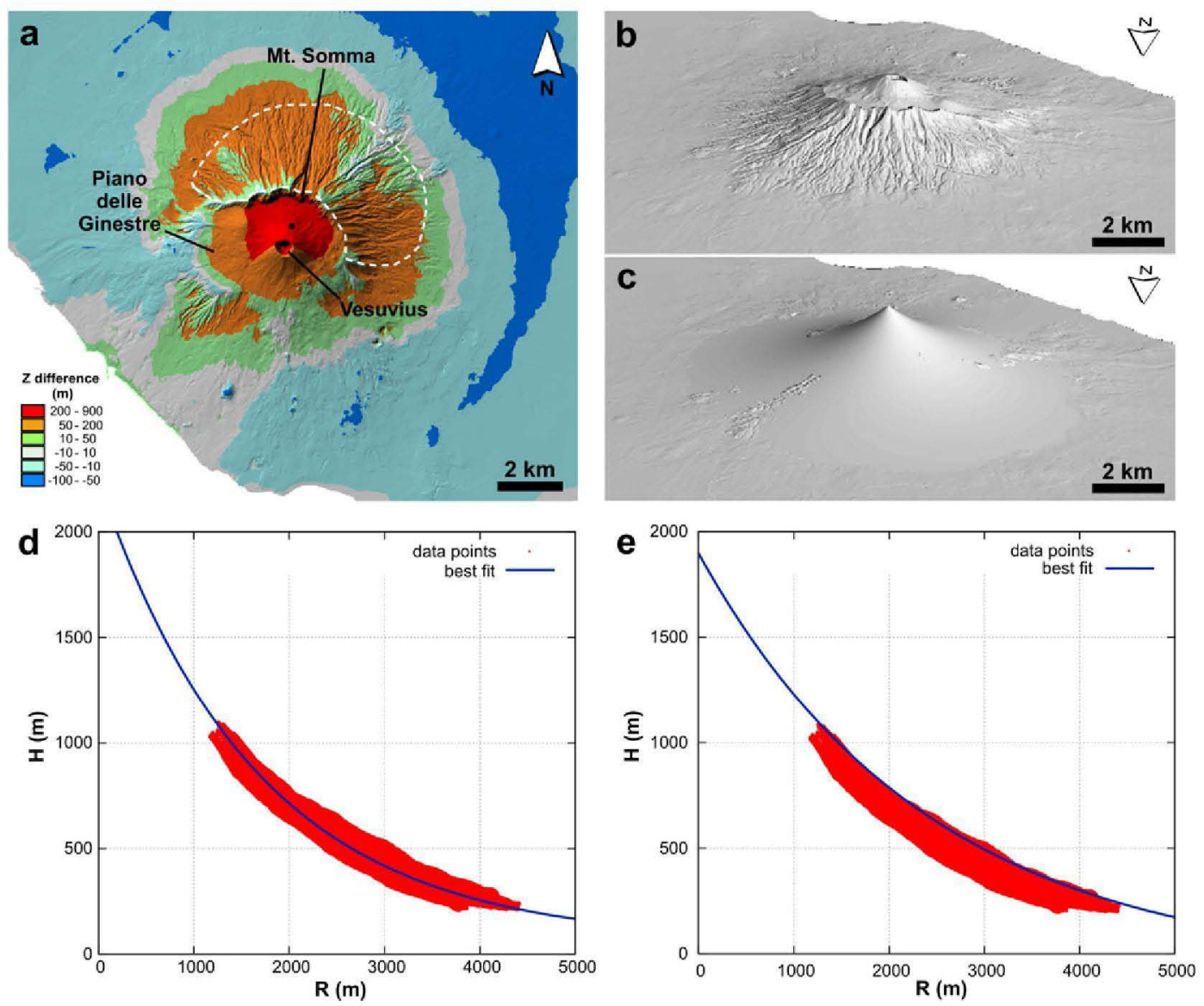

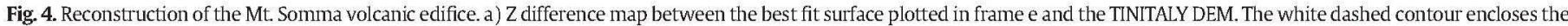

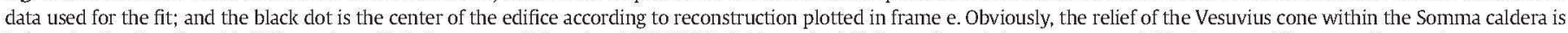

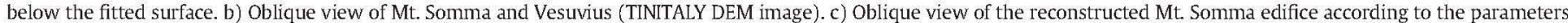

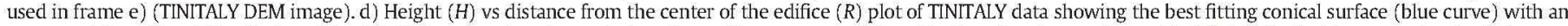

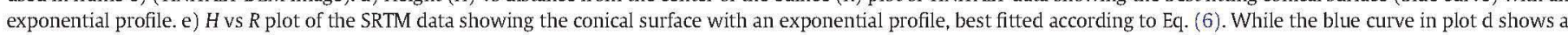

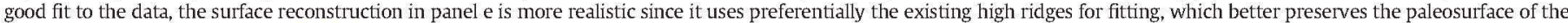
cone. See text for explanation and Table 1 for the best fit values.

in the reconstruction by giving a higher weighting to the points that are locally higher than the fitted surface. One way to do this is to define an 'ad hoc' weighting in Eq. (3). An alternative way is to minimize the following expression (which is no longer RMSE, as indicated by the asterisk):

$\operatorname{RMSE}^{*}=\sqrt{\frac{\sum_{i, j}\left[H_{i, j}-f\left(x_{i}, y_{j}, \alpha\right)\right]^{\kappa_{i, j}}}{n}}$

where $\kappa_{i j}$ is dependent on the data point and is equal to 2 if $H_{i, j}-f\left(x_{i}, y_{j}, \alpha\right)$ is negative, and 4 if it is positive. In this way if a point is at a higher elevation than the fitted surface, its contribution is squared. Fitting by minimizing Eq. (6) yields a surface that covers most of the points and allows only a small number of points to remain above the surface itself (Fig. 4e).

Such a profile, which follows the elevation points closely, defines a summit at ca. $1900 \mathrm{~m}$ a.s.l., in accordance with the maximum elevation estimate of Cioni et al. (1999). It is obvious that the lower two thirds of the original stratovolcano constitute a highly regular landform, and its shape, apart from the uppermost $800-900 \mathrm{~m}$ which collapsed repeatedly (Delibrias et al., 1979; Stothers and Rampino, 1983; Cioni et al., 1999), has been unaffected by the post- $18,000 \mathrm{yr}$ caldera evolution. This finding also implies that the $18 \mathrm{ka}$-long period of erosion was incapable of modifying the envelope of the original volcano shape, which can still be reconstructed. More importantly, our method is able to reconstruct not only highly symmetric examples such as Mayon or Semeru, but also less regular stratovolcanic edifices with apparent asymmetries.

\subsection{Mt. Cameroon}

The slope map of Mt. Cameroon (Fig. 5a) clearly shows the NE-SW elongation of the edifice, as well as the most active NE and S-SW flanks heavily dotted by hundreds of scoria and spatter cones.

To address the problem of an elongated edifice in the simplest way, the most regular (but representative) portion of the shape should be considered. In this respect, the $500 \mathrm{~m}$ contour line around $\mathrm{Mt}$. Cameroon encloses an area which is rather irregular compared to the one enclosed by the $1000 \mathrm{~m}$ contour (Fig. 5a). The topographic profile along the short axis (C-D) also shows a sudden slope change at 1000 $m$ elevation while the profile along the long axis (A-B) does not (Fig. 5b). This implies that different profile functions should be used along these two axes in order to fit the edifice down to the base. For this reason, we consider only those points higher than $1000 \mathrm{~m}$. In this way, we keep a large valley on the NW side of the volcano (named the 'Elephant Opening', Fig. 5a) but the 1700-m-high peak on the southern flank (Mt. Etinde, also known as Little Mount Cameroon, Fig. 5a) is excluded. In Fig. $5 \mathrm{c}$ we plot the height $(H)$ vs distance from the center of the edifice $(R)$ of the SRTM data along with the best fitting cone (blue curve) following exactly the same procedure as for Mayon in Fig. 1b.

The asymmetry (NE-SW elongation) of the edifice is obvious. Below $3500 \mathrm{~m}$ a.s.l., the elongation of the edifice results in an abrupt widening 

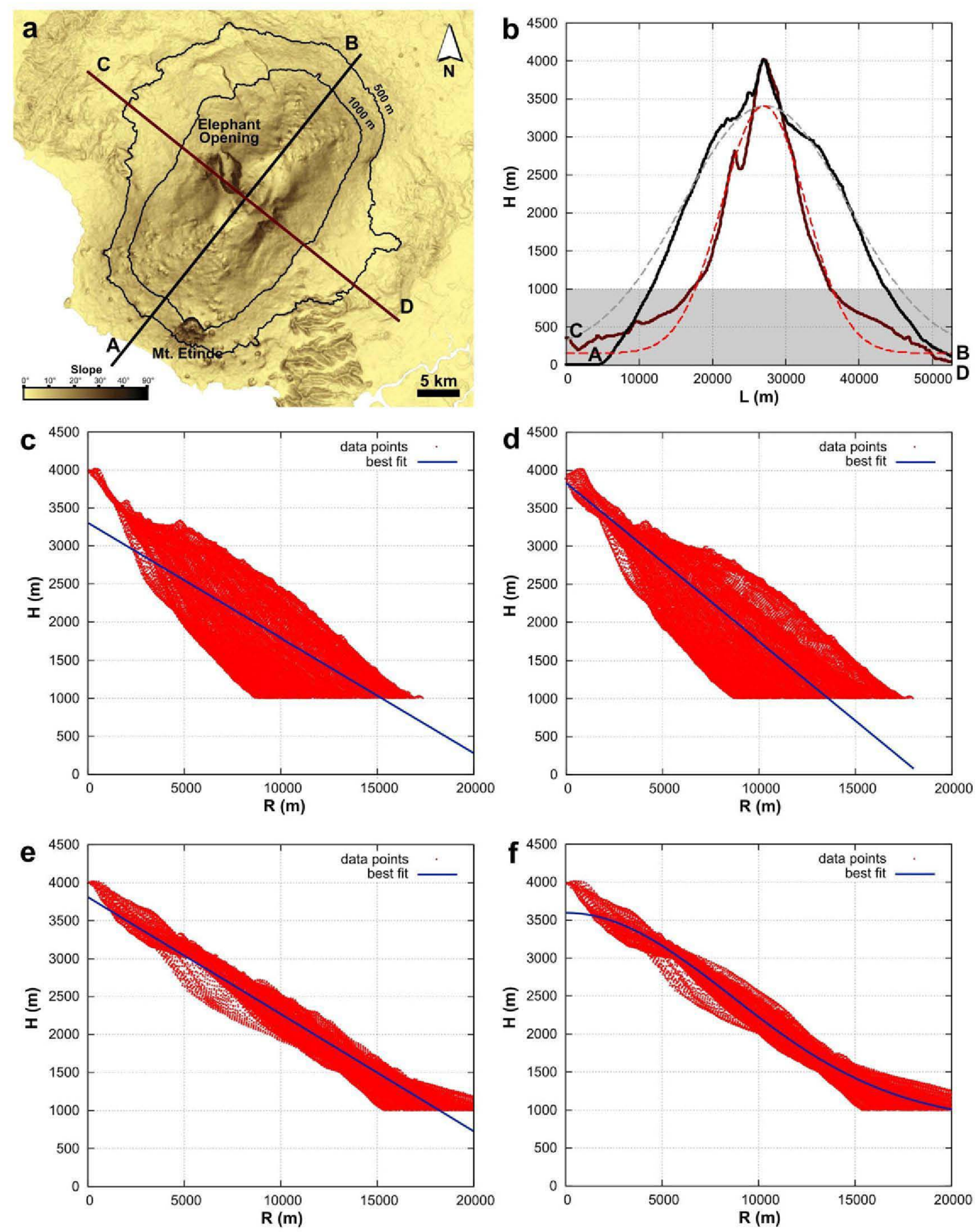

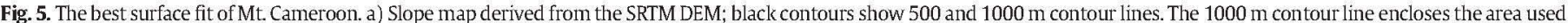

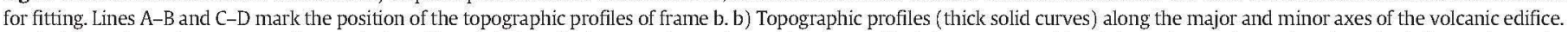

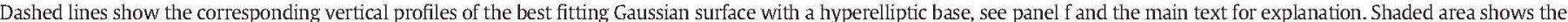

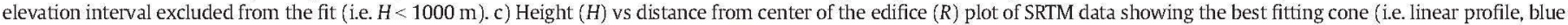

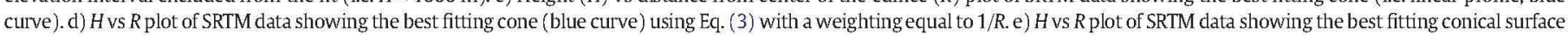

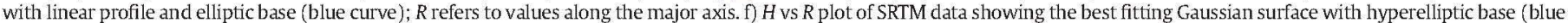
curve); $R$ refers to values along the major axis. See main text for explanation and Table 1 for best-fit values.

of the elevation points, that is, the NE and SW flanks tend to be farther from the center, while the NW and SE flanks are closer than the average distance (Fig. 6a). This trend is enhanced by the large valley of "Elephant Opening' on the NW flank, the bottom of which is closest to the center. Such an asymmetry is explained by the slow sliding of the volcano's superficial part along the long (NW and SE) flanks over the spreading basal slopes (thrusts and folds: Mathieu et al., 2011). On the contrary, the uppermost $500 \mathrm{~m}$ sector has a rather circular symmetry (i.e. elevation points evenly distributed around the center) but the overall landform is badly approximated by the fitted cone. To improve the fit in the upper part of the volcano, we also calculated the best fitting cone using Eq. (3), assigning a weighting which is inversely proportional to its distance from the edifice center to each point (Fig. 5d). As already noted for Mayon (Fig. 1d), the usage of the $1 / R$ weighting helps in fitting 
the upper part of the volcano, where there is a smaller number of points for purely geometric reasons. The $1 / R$ weighting allows an overall improvement of the fit if compared to the fit without any weighting (Fig. 5c): now the fitted surface has both lower and higher real data points at all elevations (Fig. 5d).

Next, we considered a cone with an elliptical base, and used MINUIT to find the best fitting one, i.e. to determine center, orientation, and eccentricity of the elliptical base as well as apex height and slopes of the cone (Fig. 5e). The ratio between the short axis and the long axis was 0.51 and the major diameter oriented along an azimuth angle of $39.5^{\circ}$. Just to produce a simple plot easily comparable with Fig. $5 c$, d, we rescaled the planimetric coordinates along the direction of the minor axis to the value of the major axis obtaining the plot of Fig. 5e. The effect of this transformation is that our fitting cone with the elliptic base is again simply displayed as a line, while the choice of stretching the minor axis to the major axis has the drawback of visually increasing the spreading of the points around the fitting line. Nevertheless, the data points are now much less dispersed around the best fit. The overall RMSE value associated with the fit of a regular cone is $432 \mathrm{~m}$, while the one associated with the cone with an elliptic base is only $159 \mathrm{~m}$. A more detailed comparison between the errors associated with the two fits is displayed both as maps and histograms in Fig. 6. Thus, it is clear that
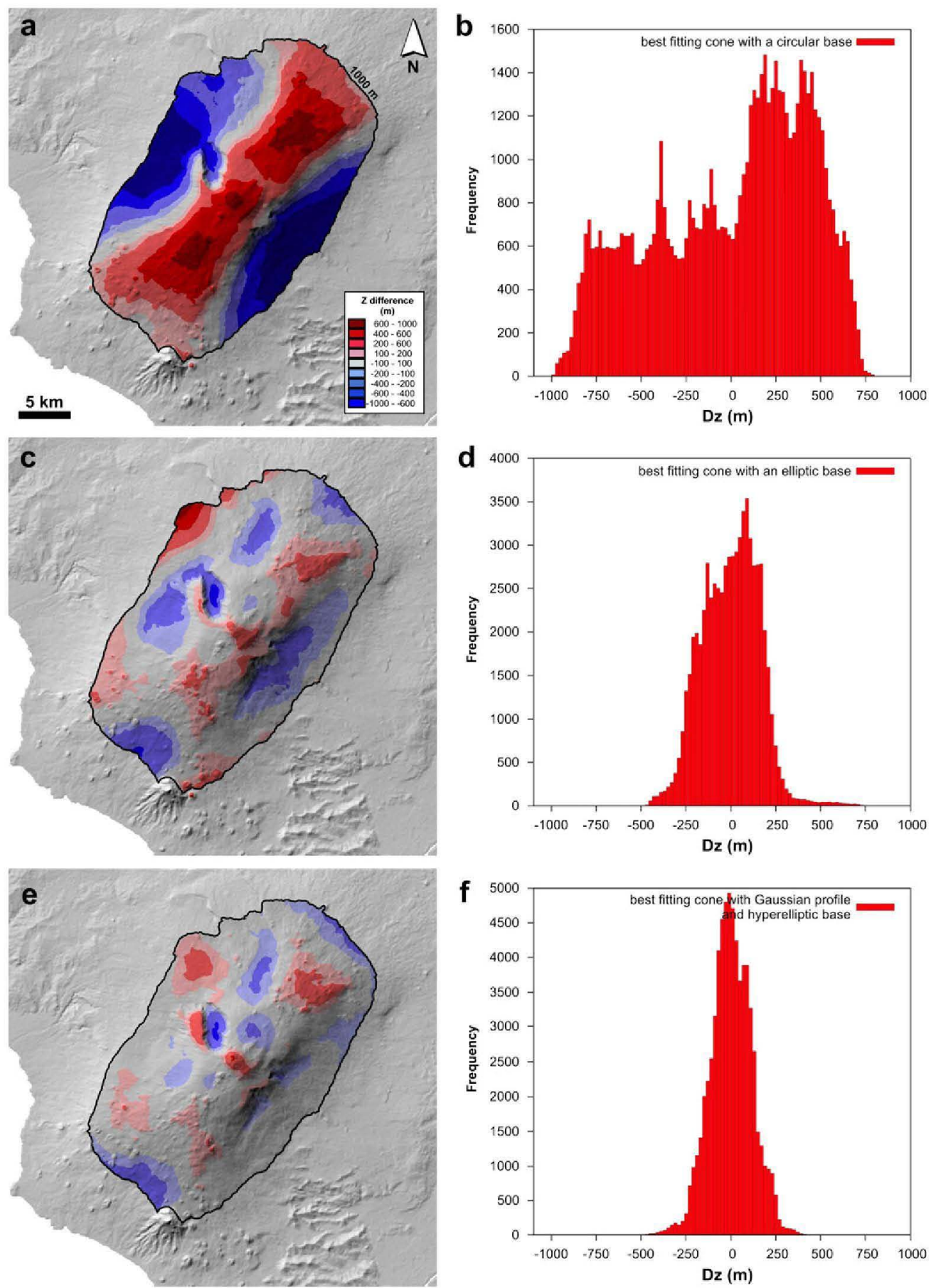

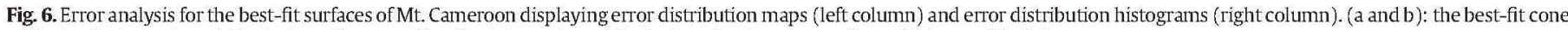
with circular base. ( $c$ and $d$ ): the best fit cone with elliptic base. (e and f): the best-fit Gaussian surface with hyperelliptic base. 
Mt. Cameroon's large-scale shape follows an ellipse, and although it is not as regular in this respect as a concentric stratovolcano, the deviations from the best-fitted ellipse are relatively low.

Fig. 7a shows the contour comparison between the SRTM DEM and the best fitting "cone" with an elliptic base: the overall shape defined by the real contours is something between an elliptic shape and a rectangular shape. For this reason we have tried to generalize the possible base of the conical surface to a superellipse, also known as the Lamé curve, defined in the Cartesian coordinate system as the set of points $(x, y)$ that satisfy:

$|x / A|^{\beta}+|y / B|^{\beta}=1$

where $A>B>0$ and $B>0$. $A$ and $B$, as in the case of the ellipse, are the major and minor axes. If $\beta$ is 2 , the curve is an ordinary ellipse (e.g. red dashed curves in Fig. 7a); if $\beta<2$, the curve is also called a hypoellipse; and if $\beta>2$, it is a hyperellipse (e.g. red dashed curves in Fig. 7b). As $\beta$ increases from 2 to $+\infty$, the curve changes in shape from an ellipse to a rectangle with sides $2 A$ and $2 B$. We have allowed $\beta$ to be a free parameter to be determined in the best fitting procedure. For example while the best fit of a cone (linear profile) with an elliptic base (i.e. $\beta$ fixed and equal to 2 ) has an RMSE value of $158.7 \mathrm{~m}$, the best fit of a cone with a superelliptic base results in a superellipse with $\beta=2.48$ ( $\beta>2$, hence a hyperelliptic base) with an RMSE value of $151.8 \mathrm{~m}$. Since we have added an extra degree of freedom to the system, a reduction in RMSE is expected and the $4 \%$ reduction is indeed very low.

We then tried to fit a cone with a power law profile:

$z=a R^{\gamma}+c$

where $\gamma$ is a constant. We have again increased the number of total parameters by one, hence a further reduction in RMSE is expected. (For $\gamma=1$ we go back to the linear profile case.) The best fitting cone with a power law profile and an elliptic base resulted in $\gamma=0.77$ for RMSE $=148.8 \mathrm{~m}$ : only a $6 \%$ decrease compared to the linear profile case. The best fitting cone with a power law profile and a generic superelliptic base resulted in an exponent $\gamma=0.80$ and $\beta=2.43$ for RMSE $=143.0 \mathrm{~m}$ : a further $4 \%$ decrease compared to the corresponding elliptic base, and a total of $10 \%$ reduction compared to the linear profile case with an elliptic base. The obtained $\gamma$ exponents $<1$ imply concave slopes for the fitting power-law profiles, which is somewhat unexpected if we compare them with the convex topographic profile along the major axis (Fig. 5b). The explanation reflects the fact that the profile along the minor axis is mostly concave and that $\gamma=0.77-0.80$ is a value range for which the power-law profile is very similar to a linear profile.

The fit of a cone with an exponential profile (Eq. (5)) and elliptic or generic superelliptic base yields RMSE values of 141.1 and $135.9 \mathrm{~m}$, respectively. Compared to the cone with an elliptic base $(R M S E=158.7 \mathrm{~m})$ we have successfully obtained a reduction of almost $14 \%$ for the exponential profile and hyperelliptic base $(\beta=2.44)$. Lastly we considered cones with a Gaussian profile (i.e. a Gaussian surface):

$z=a \exp \left(-b R^{2}\right)+c$

where $a, b$ and $c$ are parameters to be determined and only positive values of $b$ are taken into account. Fitting a Gaussian surface with an elliptic base yields RMSE $=153.8 \mathrm{~m}$, which is not an improvement. On the other hand, fitting a Gaussian surface with an generic superelliptic base gives $R M S E=116.7 \mathrm{~m}$ for $\beta=2.50$, a reduction of $26 \%$ compared to the initial fit with the linear-profile cone with an elliptic base (Figs. 5, 6 and 7). In all the examined fits with elliptic or superelliptic bases, we found very similar values for the ratio between the short and long axes, the major diameter azimuth angle and the $\beta$ exponent of Eq. (7). Fig. $6 e$ shows the discrepancies between the best fitted Gaussian surface with a hyperelliptical base and the DEM highlighting the main surface anomalies such as the summit cone and the Elephant Opening Valley on the NW side of the edifice. The missing volume in the Elephant Opening Valley seems to be balanced by an excess in volume downhill of the valley.

\section{Discussion and conclusions}

Describing and defining surface features, in particular elementary landforms, are among the main trends in geomorphometry (see Pike et al., 2009 and references therein). Within this broad topic, we have focused here on ideal (geometric) surface fits to relatively simple volcano shapes that represent different types that are known or expected to be regular. "Regular" here means that these landforms may be described by 'cones' with linear, exponential, power-law or Gaussian profiles, the base of which can be concentric, elliptic or superelliptic. Applying the MINUIT program we were able to find the best fitting geometric form in one step, albeit using a large number of input morphometric variables.
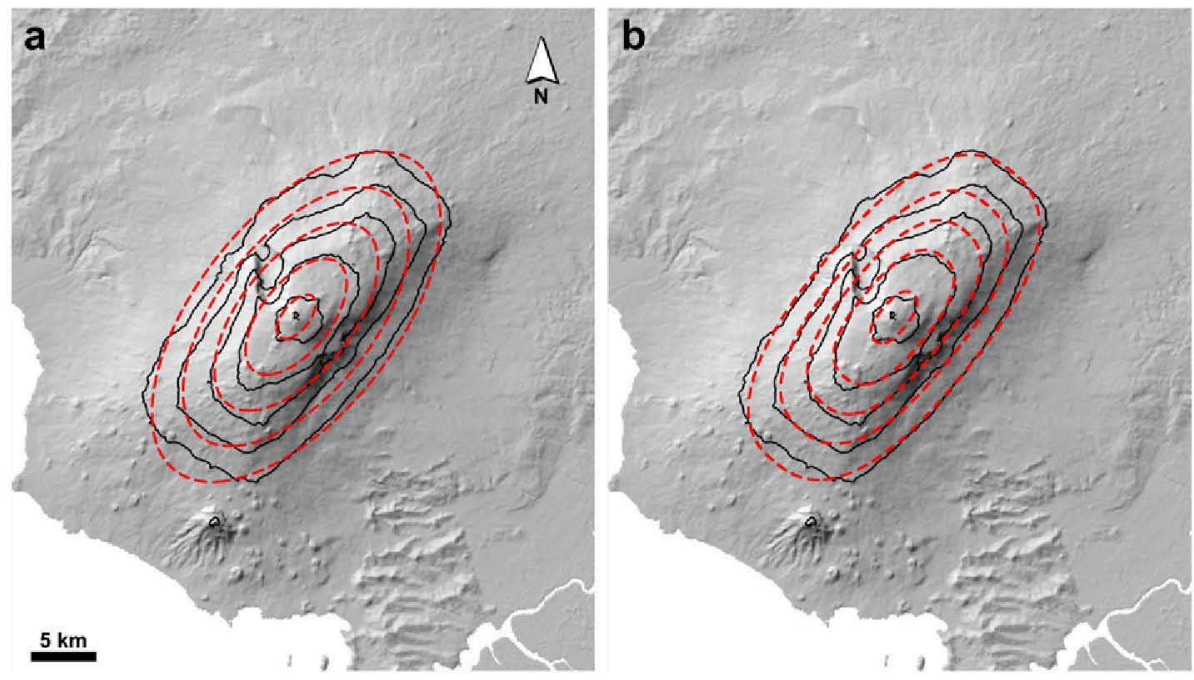

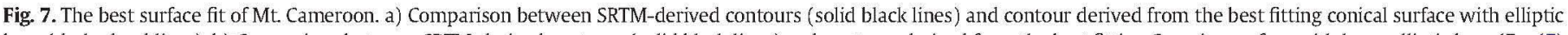

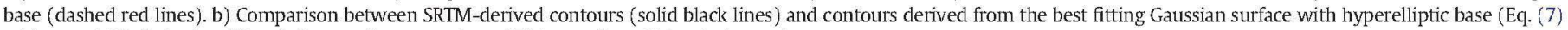
with $\alpha=2.50$, dashed red lines). Contour lines start from $1500 \mathrm{~m}$ and are $500 \mathrm{~m}$ in interval. 
The advantage of fitting a geometric surface is attractive for both theoretical and practical reasons. In theoretical terms, we can point out quantitatively to what extent a given landform is regular: if and how much it matches the expectations of having a regular, welldefined shape. In our study cases, we pointed out regular but different circular and elliptical "ideal" shapes that are attributed to long-term, combined activity from point-like and/or fissural vents. Our results imply that the activity of such vents can build up highly symmetric edifices (e.g. Mayon and Semeru) even if eruptive history causes endogenic destruction (e.g. caldera collapses at Mt. Somma). The example of Mt. Cameroon, on the other hand, demonstrates that the effect of fissure-type activity with a possible tectonic influence can modify, but not distort, the regular shape, which is best fitted by an ellipse.

To refine the theoretical approach, the stratovolcanoes of Mayon and Semeru have been fitted by regular geometric cones with minimum deviations (i.e. RMSE) from the ideal shape defined by a mathematical function. These two volcanoes, although very similar, represent two sub-types of stratovolcanoes (Karátson et al., 2010) that can be fitted either exclusively by an exponential function, or by a mixed shape, i.e. exponential and linear functions along the lower and upper parts, respectively. For the mixed shape fit of Semeru, the application of MINUIT successfully found how the two functions can be combined in order to obtain the best surface fit.

Another stratovolcanic edifice, Mt. Somma, exemplifies the benefits of surface fitting in volcano reconstruction. For a truncated or degraded volcano like Somma, whose shape is not clear, surface fitting helps to reconstruct the approximate original shape with the maximum precision possible. In our study, we were able to reconstruct the original surface using the remnant two-thirds of the volcano's flanks, despite its multiple caldera and long-term heavy erosion. The reconstruction clearly indicates that the Mt. Somma volcano is a single edifice, the shape of which is well constrained by an exponential function; moreover, it gives an original height of $1900 \mathrm{~m}$ assuming a simple, point-like summit.

The fourth example, Mt. Cameroon, is an elongated volcanic edifice, the shape of which, unlike the others, cannot be constrained successfully by fitting a concentric geometry. However, by fitting a Gaussian surface with a hyperelliptic base, we found that its surface follows the latter "ideal" shape with a relatively low error. Thus the volcano is a good example of a regular, but not circularly symmetric, edifice. We emphasize that these elongated volcano types are very numerous on Earth (e.g. rifted shields, fissure vents, tectonically controlled volcanoes and volcano groups). Moreover, this type of regularity calls attention to the fact that surface fitting may have the potential to quantitatively define "mixed" or poorly constrained landform types such as compound stratovolcanoes (cf. Grosse et al., 2009).

Fitting the "ideal" surface onto degraded volcanoes - whether circular or elliptical - also has the potential to calculate the missing volumes resulting either from endogenic processes such as sector collapse or short- to long-term erosion. However, since only active volcanoes have been included in our study, the "ideal" shape that we point out reflects mostly endogenic processes including not only the emplacement of volcanic products but also the isostatic rebound or lateral spreading, combined to a lesser extent with exogenic processes such as syn- or inter-eruptive erosion (e.g. slope redeposition) as well as compaction of loose volcanogenic deposits. On the other hand, for volcanoes which have been extinct for a long time ago, the method would be different if the aim is to reconstruct their ideal (original) shape. In the case of long-term denudation, the rate of erosion can be estimated provided the age of the volcano is known (see numerical results from the Central Andes using a similar approach by Karátson et al, (2012)).

In conclusion, our study demonstrates that the application of the MINUIT minimization library to regular-shaped volcanic landforms is highly advantageous at least for two reasons. Firstly, studying a volcano's shape can quantitatively indicate to what degree the selected landform is regular, and what type of regularity (i.e. circular or elliptical) can be defined. Fitting an ideal geometric form can be done for any sort of landform; therefore, apart from different volcano types, other regular-shaped landforms (e.g., eolian, glacial and periglacial) possessing spherical or elongated (e.g. parabolic and elliptic) geometries can also be constrained by the method. Moreover, fitting the ideal geometry might unmask surface anomalies linked to secondary morphogenetic processes - either endogenic or exogenic - that distort the geometric shape (e.g. Fig. 6e). The results, which make it possible to calculate removed volumes, can also be used for estimating longterm erosion rates of different volcano types. Likewise, erosion of nonvolcanic landforms can also be addressed.

Secondly, if we have a deeply degraded but possibly regular-shaped landform, it can be restored by the minimization program with very little error. This feature of the method is highly advantageous when we do not have evidence of the original configuration: the ideal, primary shape can then be determined by the presented method with the highest possible precision.

\section{Acknowledgments}

We are grateful to Matthieu Kervyn and an anonymous reviewer for their constructive and useful comments, as well as to Takashi Oguchi for his valuable editorial work.

\section{References}

Ahn, S.J., 2008. Geometric fitting of parametric curves and surfaces. J. Inf. Process. Syst. 4 (4), 153-158.

Castillo, P.R., Newhall, C.G., 2004. Geochemical constraints on possible subduction components in lavas of Mayon and Taal volcanoes, southern Luzon, Philippines. J. Petrol. 45 $1089-1108$.

Cioni, R., Santacroce, R., Sbrana, A., 1999. Pyroclastic deposits as a guide for reconstructing the multi-stage evolution of the Somma-Vesuvius Caldera. Bull. Volcanol. 60 207-222.

CN/ASD Group MINUT, 1993. User Guide, nProgram Library D506. CERN, Geneva.

Cotton, CA., 1952. Volcanoes as Landscape Forms, 2nd ed. Wiley \& Sons, New York, (415 pp.).

Davidson, J., Da Silva, S., 2000. Composite volcanoes. In: Sigurdsson, H. (Ed.), Encyclopedia of Volcanoes. Academic Press, New York, pp. 663-681.

Delibrias, G., di Paola, G.M., Rosi, M., Santacroce, R., 1979. La storia eruttiva del complesso vulcanico Somma-Vesuvio ricostruita dalle successioni piroclastiche del Monte Somma. Rend. Soc. Ital. Mineral. Petrol. 35, 411-438.

Deng, Y., 2007. New trends in digital terrain analysis: landform definition, representation, and classification. Prog. Phys. Geogr. 31, 405-419.

Déruelle, B., Ngounouno, I., Demaiffe, D., 2007. The "Cameroon hot line": an unique example of active alkaline intraplate structure in both oceanic and continental lithosphere C. R. Geosci. 339, 589-600

Evans, IS., 1990. General geomorphometry, In: Goudie, A.S., Anderson, M., Burt, T., Lewin, J., Richards, K., Whalley, B., Worsley, P. (Eds.), Geomorphological Techniques, 2nd edition. Unwin Hyman, London, pp. 44-56.

Fitton, J.G., 1983. Active versus passive continental rifting-evidence from the WestAfrican rift system. Tectonophysics $94,473-481$.

Fitton, J.G., Dunlop, H.M., 1985. The Cameroon line, West-Africa, and its bearing on the origin of oceanic and continental alkali basalt. Earth Planet. Sci. Lett. 72, 23-38.

Fornaciai, A., Favalli, M., Karátson, D., Tarquini, S., Boschi, E., 2012. Morphometry of scoria cones, and their relation to geodynamic setting: a DEM-based analysis. J. Volcanol. Geotherm. Res. 217/218, 56-72.

Gercek, D., Toprak, V., Strobl, J., 2011. Object-based classification of landforms based on their local geometry and geomorphometric context. Int. J. Geogr. Inf. Sci. 25 1011-1023.

Grosse, P., van Wyk de Vries, B., Petrinovic, I.A., Euillades, P.A., Alvarado, G.E., 2009 Morphometry and evolution of arc volcanoes. Geology 37, 651-654.

Hermann, T., Kovács, Z., Várady, T., 1997. Special applications in surface fitting. In Strasser, W., Klein, R., Rau, R. (Eds.), Geometric Modeling: Theory and Practice. Springer, Berlin, pp. 14-31.

James, F. Winkler, M., 2004. Minuit User's Guide. CERN, Geneva

Karátson, D., Thouret, J.C., Moriya, I., Lomoschitz, A., 1999. Erosion calderas: origins, processes, structural and climatic control. Bull. Volcanol. 61, 174-193.

Karátson, D., Favalli, M., Tarquini, S., Fornaciai, A., Wörner, G., 2010. The regular shape of stratovolcanoes: a DEM-based morphometrical approach. J. Volcanol. Geotherm. Res. 193, 171-181.

Karåtson, D., Telbisz, T., Wörner, G., 2012. Erosion rates and erosion patterns of Neogene to Quatemary stratovolcanoes in the Western Cordillera of the Central Andes: an SRTM DEM based analysis. Geomorphology 139 (140), 122-135.

Lavigne, F., 2004. Rate of sediment yield following small-scale volcanic eruptions: a quantitative assessment at the Merapi and Semeru stratovolcanoes, Java, Indonesia. Earth Surf. Process. Landf. 29, 1045-1058. 
Marzoli, A., Piccirillo, E.M., Renne, P.R., Bellieni, G., lacumin, M., Nyobe, J.B., Tongwa, A.T., 2000. The Cameroon line revisited: petrogenesis of continental basaltic magmas from lithospheric and asthenospheric mantle sources. J. Petrol. 41, 87-109.

Mathieu, L., Kervyn, M., Ernst, G.G.J., 2011. Field evidence for flank instability, basal spreading and volcano-tectonic interactions at Mt Cameroon, West Africa. Bull. Volcanol. 73, 851-867.

Pike, R.J., Evans, I.S., Hengl, T., 2009. Geomorphometry: a brief guide. In: Hengl, T., Reuter H.I. (Eds.), Geomorphometry - Concepts, Software, Applications. Developments in Soil Science, vol. 33. Elsevier, Amsterdam, pp. 3-30.

Rabus, B., Eineder, M., Roth, A., Bamler, R., 2003. The shuttle radar topography mission - a new class of digital elevation models accuired by spaceborne radar. ISPRS J. Photogramm. Remote Sens. 57, 241-262.
Ramos-Villarta, S.C., Corpuz, E.G., Newhall, C.G., 1985. Eruptive history of Mayon volcano, Philippines. Philipp. J. Volcanol. 2, 1-35.

Stothers, R.B., Rampino, M.R., 1983. Volcanic eruptions in the Mediterranean before AD 630 from written and archaeological sources. J. Geophys. Res. 88, 6357-6371.

Tarquini, S., Isola, I., Favalli, M., Mazzarini, F., Bisson, M., Pareschi, M.T., Boschi, E., 2007. TINITALY/01: a new triangular irregular network of Italy. Ann. Geophys. 50, 407-425.

Tarquini, S., Vinci, S., Favalli, M., Doumaz, F., Fornaciai, A., Nannipieri, L., 2012. Release of a $10 \mathrm{~m}$-resolution DEM for the Italian territory: comparison with global coverage DEMs and anaglyph-mode exploration via the web. Comput. Geosci. 38, 168-170.

Thouret, J.C. 1999. Volcanic geomorphology - an overview. Earth Sci. Rev. 47, 95-131. Wood, C.A., 1980. Morphometric evolution of cinder cones. J. Volcanol. Geotherm. Res. 7, $387-413$. 